\title{
The Four-Dimensional Fluid Space Interpretation of Quantum Mechanics
}

\author{
Kennard Callender \\ Universidad Tecnológica de Panamá \\ Centro Regional de Veraguas
}

\begin{abstract}
An interpretation of quantum mechanics is formulated based on the hypothesis that physical space is a four-dimensional incompressible inviscid fluid. This hypothesis is used to derive the one-dimensional Schrödinger equation from the equations for two-dimensional incompressible irrotational inviscid fluid flow.
\end{abstract}

\section{Contents}

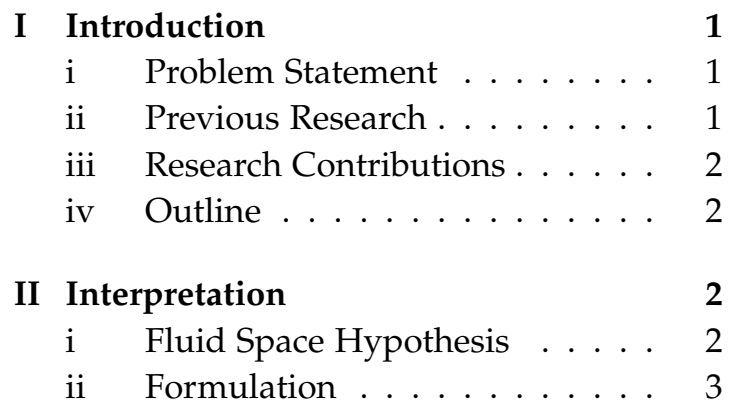

III The Hamilton-Jacobi Equation 5 i One-Dimensional HJE ...... 5

ii Complex Conjugate HJE . . . . . 6

iii Physical Interpretation . . . . . . 7

\begin{tabular}{ll}
\hline IV Derivation & 7
\end{tabular}

i Momentum Equation. . . . . . . 8

ii Schrödinger's Equation . . . . . 8

V Conclusion

9

\section{INTRODUCTION}

$\mathrm{T}$ HE mathematical formalism of quantum mechanics has held up to rigorous tests in a broad range of experiments. The interpretation of this formalism, however, is a subject of unprecedented dissension. A realistic deterministic local hidden-variable interpretation of quantum mechanics would be a strong candidate to bring consensus, but Bell's theorem implies such an interpretation is not possible. An in-depth discussion of this issue, including a comprehensive review of interpretations of quantum mechanics from a historical perspective, is given by Max Jammer [1].

\section{i. Problem Statement}

In the present paper, we formulate the FourDimensional Fluid Space Interpretation of Quantum Mechanics. It is a realistic deterministic local hidden-variable theory based on the hypothesis that physical space is a four-dimensional incompressible inviscid fluid. In order to prove it is mathematically consistent and valid, we derive the one-dimensional Schrödinger equation from the equations for two-dimensional incompressible irrotational inviscid fluid flow. We argue that Bell's theorem does not take into account particle-fluid systems, therefore it is not applicable to the interpretation presented here.

\section{ii. Previous Research}

The idea that space could be four-dimensional was first suggested by Gunnar Nordström in 
1914, and a few years later by Theodor Kaluza. Nordström developed a theory of gravity that introduced an additional space dimension [2]. In his theory, the additional dimension provided coupling to electromagnetism. Kaluza unified Einstein's general relativity with electromagnetism by using a five-dimensional manifold (four dimensions of space and one of time) [3]. These unifications of gravity and electromagnetism, that assume there are four space dimensions instead of three, provide sufficient incentive to explore the four-dimensional space hypothesis further.

The other important result that motivates the research presented here was the one obtained by Erwin Madelung in 1926. He derived Schrödinger's equation from the hydrodynamic equations for an irrotational inviscid flow [4]. Even though Madelung did not identify physical space as the fluid in his interpretation, the derivation shows there exists a relation between Schrödinger's equation and the equations of an inviscid fluid undergoing irrotational flow.

\section{iii. Research Contributions}

As mentioned in section 1.2, the fourdimensional space hypothesis, and the connection between the hydrodynamic equations and quantum mechanics, have been proposed before. However, to the best of the author's knowledge, the following are original contributions from the present article:

- The Four-Dimensional Fluid Space Interpretation of Quantum Mechanics.

- A nonrigorous argument justifying why physical space can be described by the mathematical model of an incompressible inviscid fluid.

- Formulation and physical interpretation of the complex conjugate Hamilton-Jacobi equation.

- Derivation of Schrödinger's equation from the complex conjugate Hamilton-Jacobi equation.

\section{iv. Outline}

The outline of this paper is as follows. The Four-Dimensional Fluid Space Interpretation of Quantum Mechanics is formulated in Section 2. The section starts with an argument that supports the incompressible inviscid fluid space hypothesis. Then, the postulates about the nature of time, physical space, and elementary particles are stated. Finally, the interpretation of the mathematical formalism of quantum mechanics is presented.

A complex version of the Hamilton-Jacobi equation (HJE) is developed in Section 3. After providing a brief review of the onedimensional HJE, the complex conjugate HJE is defined, and a physical interpretation of what it describes is given. It is shown that the complex conjugate HJE represents a particle moving along the streamlines of an incompressible irrotational inviscid fluid flow in two-dimensional space.

Section 4 contains the derivation of the onedimensional Schrödinger equation from the complex conjugate HJE. This derivation provides evidence that the interpretation proposed here is mathematically consistent and valid. The apparently conflicting issues with Bell's theorem are addressed in the Conclusion section.

\section{INTERPRETATION}

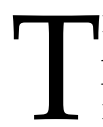
HE Four-Dimensional Fluid Space Interpretation of Quantum Mechanics is formulated in this section. Because the fluid space hypothesis is of fundamental importance to the theory, a nonrigorous argument is provided to justify it. After that, we state the proposed postulates about the nature of time, physical space, and elementary particles. Based on these postulates, we present the interpretation of the mathematical formalism of quantum mechanics.

\section{i. Fluid Space Hypothesis}

The fluid space hypothesis proposes that physical space is an incompressible inviscid fluid. 
We proceed to present a logical argument to justify it. The argument is not rigorous, but it provides valid reasons to take the hypothesis seriously. By physical space, we mean the region of space that does not include matter. So according to this definition, massive elementary particles move within physical space, but are not part of it. The term physical space will be used throughout this paper in the manner defined here.

We start by assuming physical space is a continuum, and thus can be described by one of the mathematical models in the field of continuum mechanics. In the following argument (and throughout this paper), the words fluid, rigid solid, and deformable solid, refer to these models.

- Physical space satisfies the continuum condition given by the field of continuum mechanics. Hence, it must be a fluid, a rigid solid, or a deformable solid.

- The mathematical model of a rigid solid does not allow objects to move within it. Likewise, the mathematical model of a deformable solid does not allow objects to move within it without offering some resistance.

- Physical space allows elementary particles to move within it without any resistance. Therefore, it cannot be a rigid or a deformable solid. It must be a fluid.

- The volume of physical space is always constant. Consequently, it must be an incompressible fluid.

- Physical space offers no resistance to objects moving within it. Thus, it must be an inviscid fluid.

From these premises, it follows that physical space is an incompressible inviscid fluid.

\section{ii. Formulation}

The Four-Dimensional Fluid Space Interpretation of Quantum Mechanics is a realistic deterministic local hidden-variable theory. It is based on the following postulates about the nature of time, physical space, and elementary particles:
- Time is absolute.

- Physical space is four-dimensional.

- Physical space is an incompressible inviscid fluid.

- The fluid flow of physical space is irrotational.

- Elementary particles always have a definite position and their movement is completely deterministic.

- Elementary particles are directly influenced only by their immediate surroundings.

- Elementary particles move along the streamlines of the fluid flow of physical space.

- Based on their perception at the macroscopic level, observers assume physical space is three-dimensional, and this assumption has unexpected effects on the measurements they perform.

According to these postulates, the flow of physical space can be described by streamlines and velocity vectors tangent to them. Elementary particles move along those streamlines and their velocity is the velocity tangent to the streamline at the point of the particle's location. The particle always has a definite position, regardless if it is being measured or not, and its movement is completely deterministic. Additionally, the particle is affected only by it's immediate surroundings. In other words, this is a local theory. Also, because the flow of physical space is incompressible, irrotational, and inviscid; potential theory is applicable, and superposition of basic flows generates more complicated flows.

The last postulate listed, has important consequences that result in two mathematical conditions. We shall refer to that postulate as the observer's assumption, and to the conditions as the first and second mathematical conditions of the observer's assumption. They are as follows:

- Because the observer assumes space is three-dimensional, he only assigns values to the $x-y-z$ components of any measured observable that requires them. This effec- 
tively makes the fourth component of the observable (which we shall refer to as the $w$ component) equal to zero.

- Even though the particle is moving in fourdimensional space, the observer assumes its position only has three components, effectively setting the $w$ component of the particle's position equal to zero. Thus, as time evolves, the four-dimensional frame of reference rotates in such a way that the $w$-axis is always perpendicular to the particle's position.

These two conditions are necessary to derive Schrödinger's equation from the complex conjugate Hamilton-Jacobi equation.

Based on these postulates, we proceed to interpret the wave function formulation of quantum mechanics. One of the fundamental axioms of quantum mechanics states that for every physical observable there corresponds a linear operator which possesses a complete set of orthonormal eigenvectors and a corresponding set of real eigenvalues. We claim these eigenvectors and their superpositions are mathematical objects that describe and represent incompressible irrotational inviscid fluid flows, not particles.

Even though the eigenvectors from quantum mechanics describe fluid flows, they can be used to obtain indirect information about particles. This is because elementary particles move along the streamlines of the flow of physical space, and their velocity is the velocity tangent to the streamline at the point of the particle's position. So we have that the eigenvectors corresponding to a particular physical observable of a particle, describe and represent flows where that observable has the same value for every single point where the particle could be at. For example, the eigenvectors corresponding to the momentum operator, describe and represent flows of constant velocity (uniform flows) that are multipied by the mass of the particle. Therefore, a particular eigenvector of the momentum operator will describe a particle moving in a flow where it will have the same momentum for every single point where it could be found.
The operators, eigenvectors, and eigenvalues of quantum mechanics are mathematical tools used to represent the measurement process of a system. The operators specify which observable is been measured, the eigenvectors describe flows where the value of an observable is constant, and the eigenvalues provide the value obtained from taking the measurement. The reason these eigenvalues are required to be real is because of the first mathematical condition of the observer's assumption. That condition forces the fourth component of any observable to be equal to zero, and in the mathematical formalism of quantum mechanics, that component corresponds to the imaginary part of the eigenvalues. Similarly, because of the second mathematical condition of the observer's assumption, the eigenvectors are functions of only three spatial coordinates (i.e., $x, y$, and $z)$. This means the $w$-axis of the observer's frame of reference is always perpendicular to the particle's position.

The orthodox interpretation of quantum mechanics asserts that the state of a system is completely described by the wave function. We claim this is not true. The wave function only describes incompressible irrotational inviscid fluid flows that, at any given instant in time, provide incomplete information about the system. The wave function can be written in terms of superpositions of eigenvectors corresponding to any required physical observable. The coefficients in the superposition make it possible to obtain indirect information about the particle, because they dictate how probable each quantum state is. This explains why superposition of quantum states makes physical sense: physical space is in a superposition of flows, while the particle (which is moving along a streamline of the flow) is only in one of the possible states at any given instant in time.

Once a measurement is taken, the wave function collapses to a particular eigenvector of the observable. In doing so, our knowledge of that observable is updated, but (necessarily) other information about the system is lost. This occurs because the wave function always describes fluid flows, not particles. 
The collapse process reveals that the wave function does not necessarily represent the actual flow of physical space. For example, when a measurement of momentum is taken, the initial wave function collapses, and the resulting wave function will be equal to a particular eigenvector of momentum. This means the particle has that momentum at that instant, but the actual flow of physical space does not necessarly has to be a uniform flow. Only when the potential energy function of a system and the appropriate boundary conditions are specified, is the actual flow of physical space accurately described. All this leads to the conclusion that the collapse of the wave function is the result of the observer's unawareness that he is dealing with a four-dimensional particle-fluid system.

Schrödinger's equation describes the time evolution of the particle-fluid system. The potential energy function in the Hamiltonian operator specifies the streamlines of the actual flow of physical space. The total energy of the particle moving along those streamlines is fixed by the energy eigenvalue. If the initial position of the particle was also provided, then its position and momentum could be simultaneously determined at any time. Nevertheless, the mathematical formalism of quantum mechanics does not directly specify the particle's initial position, which makes the theory an incomplete description of reality.

The Dirac delta function can be used to indirectly specify the particle's initial position, but using this function comes at a cost. The information about the particle's total energy is lost, which means the position and momentum of the particle can never be known simultaneously. Similarly, a momentum eigenvector can be used to specify the initial momentum of the particle, but its position would then be unknown because eigenvectors describe fluid flows. In conclusion, Schrödinger's equation dictates how the wave function evolves in time, this evolution is in accordance with the actual flow of physical space which is specified through the potential energy function, and the lack of a proper initial condition makes the formulation incomplete.

\section{The Hamilton-Jacobi Equation}

$\mathrm{T}$ HE complex conjugate Hamilton-Jacobi equation is developed in this section. It is shown that the physical interpretation of this equation corresponds to a particle moving along the streamlines of an incompressible irrotational inviscid fluid flow in two-dimensional space. From this formulation, we can derive the one-dimensional Schrödinger equation, and thus prove that the Four-Dimensional Fluid Space Interpretation of Quantum Mechanics is mathematically consistent and valid. A brief overview of the onedimensional Hamilton-Jacobi equation is given first.

\section{i. One-Dimensional HJE}

We begin by providing a review of onedimensional Hamiltonian mechanics and of the one-dimensional Hamilton-Jacobi equation. For simplicity, the system considered here is that of a single particle moving on a straight line under the influence of a conservative force that depends only upon the spatial coordinates.

One-dimensional Hamiltonian mechanics is a formulation of classical mechanics that uses parameters $(q, p, t) \in \mathbb{R} \times \mathbb{R} \times \mathbb{R}$, where $q$ is the generalized position, $p$ is the conjugate momentum, and $t$ is time. The Hamiltonian function $H: \mathbb{R} \times \mathbb{R} \rightarrow \mathbb{R}$ completely represents the constraints of the system via Hamilton's equations, given by

$$
\begin{gathered}
\frac{d p}{d t}=-\frac{\partial H(q, p)}{\partial q} \\
\frac{d q}{d t}=\frac{\partial H(q, p)}{\partial p}
\end{gathered}
$$

In Cartesian coordinates, the Hamiltonian function for a particle of mass $m$ under the influence of a conservative force is

$$
H(q, p)=\frac{p^{2}}{2 m}+V(q)+C
$$

where $V: \mathbb{R} \rightarrow \mathbb{R}$ is the potential energy function and $C$ is an arbitrary real constant. Usually, the constant $C$ is taken to be equal to zero, but it can take any other convenient value. If the 
potential energy function depends only upon the spatial coordinates, then the total energy of the system is conserved and is equal to the Hamiltonian. So we have

$$
H(q, p)=E
$$

where $E$ is a positive real constant equal to the total energy of the system.

The one-dimensional Hamilton-Jacobi equation is a reformulation of classical mechanics, equivalent to Hamiltonian mechanics. It is a first order, nonlinear partial differential equation given by

$$
H\left(q, \frac{\partial S(q, t)}{\partial q}\right)+\frac{\partial S(q, t)}{\partial t}=0
$$

where $H: \mathbb{R} \times \mathbb{R} \rightarrow \mathbb{R}$ is the Hamiltonian function, $S: \mathbb{R} \times \mathbb{R} \rightarrow \mathbb{R}$ is Hamilton's principal function, $q \in \mathbb{R}$ is the generalized position, and $t \in \mathbb{R}$ is time. In this formulation, the conjugate momentum $p \in \mathbb{R}$ corresponds to

$$
p=\frac{\partial S(q, t)}{\partial q}
$$

For the case considered here, the Hamiltonian does not depend explicitly on time, therefore Hamilton's principal function can be written in the following form

$$
S(q, t)=W(q)-E t
$$

where $W: \mathbb{R} \rightarrow \mathbb{R}$ is Hamilton's characteristic function, and $E$ is a positive constant equal to the total energy of the system. From eqs. 6 and 7 , we can see that the conjugate momentum $p$ is a function of $q$ alone.

The HJE can be derived from Hamiltonian mechanics. This can be done by treating Hamilton's principal function as the generating function for a canonical transformation of the Hamiltonian function.

\section{ii. Complex Conjugate HJE}

Next, we formulate a complex analytic version of the Hamilton-Jacobi equation. This is done by replacing all the real-valued parameters and functions of the one-dimensional HJE with complex-valued parameters and functions (except for time). For mathematical reasons, instead of using $q \in \mathbb{C}$ to represent the generalized position on a plane, we use its complex conjugate $\bar{q} \in \mathbb{C}$. Because of this, we shall call this formulation the complex conjugate HJE.

We start by defining the complex Hamiltonian function $H: \mathbb{C} \times \mathbb{C} \rightarrow \mathbb{C}$, which completely specifies the constraints of the system via complex versions of Hamilton's equations, given by

$$
\begin{gathered}
\frac{d p}{d t}=-\frac{\partial H(\bar{q}, p)}{\partial \bar{q}} \\
\frac{d \bar{q}}{d t}=\frac{\partial H(\bar{q}, p)}{\partial p}
\end{gathered}
$$

where $\bar{q} \in \mathbb{C}$ is the generalized position on a plane, $p \in \mathbb{C}$ is the conjugate momentum on a plane, and $t \in \mathbb{R}$ is time. In Cartesian coordinates, the complex Hamiltonian function for a particle of mass $m$ under the influence of $V$ is given by

$$
H(\bar{q}, p)=\frac{p^{2}}{2 m}+V(\bar{q})+C
$$

where $V: \mathbb{C} \rightarrow \mathbb{C}$ is the complex potential energy function and $C$ is an arbitrary complex constant. Similarly to the previous case, if the complex potential energy function depends only upon the spatial coordinates, then the total energy of the system is conserved and we can set it equal to the complex Hamiltonian. So we have

$$
H(\bar{q}, p)=E
$$

where $E$ is a positive real constant equal to the total energy of the system.

The complex conjugate Hamilton-Jacobi equation is a first order, nonlinear partial differential equation, defined by

$$
H\left(\bar{q}, \frac{\partial S(\bar{q}, t)}{\partial \bar{q}}\right)+\frac{\partial S(\bar{q}, t)}{\partial t}=0
$$

where $H: \mathbb{C} \times \mathbb{C} \rightarrow \mathbb{C}$ is the complex Hamiltonian function, $S: \mathbb{C} \times \mathbb{R} \rightarrow \mathbb{C}$ is Hamilton's complex principal function, $\bar{q} \in \mathbb{C}$ is the generalized position on a plane, and $t \in \mathbb{R}$ is time. 
The conjugate momentum on a plane, $p \in \mathbb{C}$, corresponds to

$$
p=\frac{\partial S(\bar{q}, t)}{\partial \bar{q}}
$$

For the case considered here, the complex Hamiltonian does not depend explicitly on time, therefore Hamilton's complex principal function can be written as

$$
S(\bar{q}, t)=W(\bar{q})-E t
$$

where $W: \mathbb{C} \rightarrow \mathbb{C}$ is Hamilton's complex characteristic function, and $E$ is a positive constant equal to the total energy of the system. As can be seen from eqs. 13 and 14, the conjugate momentum on a plane, $p$, is a function of $\bar{q}$ alone.

Like the HJE, the complex conjugate HJE can be derived by treating Hamilton's complex principal function as the generating function for a canonical transformation of the complex Hamiltonian function.

\section{iii. Physical Interpretation}

Finally, we proceed to give a physical interpretation of the complex conjugate HamiltonJacobi equation. Consider a particle of mass $m$, moving on a two-dimensional plane. Let's represent the position of the particle and its momentum with

$$
\bar{q}=x-i w
$$

and

$$
p=p_{x}+i p_{w}
$$

respectively. The variables $x, w, p_{x}$, and $p_{w}$ are all elements of the real numbers. By substituting eq. 14 into eq. 13 we get

$$
p=\frac{d W}{d \bar{q}}
$$

Because $W$ is a function of $\bar{q}$ alone, it is analytic with respect to it. This means the momentum $p$ is also analytic with respect to $\bar{q}$, and thus it satisfies the Cauchy-Riemman conditions corresponding to $\bar{q}$, which are

$$
\frac{\partial p_{x}}{\partial x}=-\frac{\partial p_{w}}{\partial w}
$$

$$
\frac{\partial p_{w}}{\partial x}=\frac{\partial p_{x}}{\partial w}
$$

Dividing eqs. 18 and 19 by the mass of the particle, and moving all the terms to the left gives

$$
\begin{aligned}
& \frac{\partial\left(p_{x} / m\right)}{\partial x}+\frac{\partial\left(p_{w} / m\right)}{\partial w}=0 \\
& \frac{\partial\left(p_{w} / m\right)}{\partial x}-\frac{\partial\left(p_{x} / m\right)}{\partial w}=0
\end{aligned}
$$

Given that $p_{x} / m$ and $p_{w} / m$ are the $x$ and $w$ components of the particle's velocity, and realizing eqs. 20 and 21 correspond to the conditions of an incompressible irrotational fluid flow in two-dimensional space, we can conclude that the particle is moving along the streamlines of that type of flow.

The potential energy function of the complex Hamiltonian (given by eq. 10) corresponds to a conservative force. Therefore, there is no friction resisting the movement of the particle, which means the fluid flow is inviscid. Thus, the equations from the complex conjugate Hamilton-Jacobi equation (eqs. 12 and 13) describe a particle of mass $m$ moving along the streamlines of an incompressible irrotational inviscid fluid flow in two-dimensional space.

Hamilton's complex characteristic function, $W$, is equal to the product of the conjugate of the flow's complex potential and the mass of the particle. This function describes the streamlines and the equipotentials of the flow. Hamilton's complex principal function, $S$, contains all the necessary information about the particle-fluid system, except for the actual position of the particle. An initial condition of the particle's position needs to be specified so that the system is completely described.

\section{Derivation}

$\mathrm{I}$ $\mathrm{N}$ this section we derive the momentum eigenvalue equation and Schrödinger's equation from the complex conjugate Hamilton-Jacobi equation. All the variables and functions not defined here, correspond to the ones defined in section 3.2 and 3.3. 
The system we are describing is that of a single particle of mass $m$ moving along the streamlines of an incompressible irrotational inviscid fluid flow in two-dimensional space. We use the complex plane to represent the $x$ and $w$ components of the position and momentum observables (or any other observable that is a function of them), where the $x$ components corresponds to the real axis, and the $w$-components corresponds to the imaginary axis. The position and momentum of the particle are given by

$$
\bar{q}=x-i w
$$

and

$$
p=p_{x}+i p_{w}
$$

respectively. The variables $x, w, p_{x}$, and $p_{w}$ are all elements of the real numbers. The complex wave function is defined by

$$
\Psi(\bar{q}, t)=e^{i S / \hbar}
$$

where $\Psi: \mathbb{C} \times \mathbb{R} \rightarrow \mathbb{C}, S: \mathbb{C} \times \mathbb{R} \rightarrow \mathbb{C}$ is Hamilton's complex principal function, and $\hbar$ is the reduced Planck's constant. This function contains all the information about the particlefluid system, except for the particle's position at any given time $t$. In other words, the particle is definitely at some particular point on the plane, moving along the streamlines of the fluid flow, but the complex wave function (as defined by eq. 24) does not tell us where.

The observer's assumption (described in section 2.2) has two consequences that can be expressed as mathematical conditions. The first mathematical condition forces the fourth component of an observable to be equal to zero. The fourth component of an observable corresponds to the imaginary part of the eigenvalue, therefore, eigenvalues must be real. The second mathematical condition effectively sets the $w$ component of the particle's position equal to zero. Hence we have

$$
w=0
$$

This last condition is equivalent to evaluating the eigenvector along the $x$-axis.

\section{i. Momentum Equation}

We start by deriving the momentum eigenvalue equation in the wavefunction formulation of quantum mechanics. From eqs. 24 and 13 we have

$$
\begin{aligned}
-i \hbar \frac{\partial \Psi}{\partial \bar{q}}=-i \hbar \frac{\partial e^{i S / \hbar}}{\partial \bar{q}}=\frac{\partial S}{\partial \bar{q}} e^{i S / \hbar}=p \Psi \\
-i \hbar \frac{\partial \Psi}{\partial \bar{q}}=p \Psi
\end{aligned}
$$

The first condition of the observer's assumption states that we should substitute the momentum $p$ with the real constant $p_{o}$, therefore

$$
-i \hbar \frac{\partial \Psi}{\partial \bar{q}}=p_{o} \Psi
$$

Applying the second condition of the observer's assumption (eq. 25) gives

$$
-i \hbar \frac{\partial \Psi}{\partial x}=p_{o} \Psi
$$

which is the momentum eigenvalue equation of quantum mechanics.

\section{ii. Schrödinger's Equation}

We proceed to derive Schrödinger's equation in the position basis. From eqs. 24 and 12 we get

$$
\begin{aligned}
i \hbar \frac{\partial \Psi}{\partial t}=i \hbar \frac{\partial e^{i S / \hbar}}{\partial t} & =-\frac{\partial S}{\partial t} e^{i S / \hbar}=H \Psi \\
i \hbar \frac{\partial \Psi}{\partial t} & =H \Psi
\end{aligned}
$$

The complex potential function depends only upon the spatial coordinates, which means eq. 11 can be used. Therefore, substituting eq. 11 into eq. 29, and applying the second condition of the observer's assumption (eq. 25) gives

$$
i \hbar \frac{\partial \Psi}{\partial t}=E \Psi
$$

This is the time dependent component of Schrödinger's equation. Because eq. 30 was evaluated along the $x$-axis, the wave function, $\Psi$, is only a function of $x$ and $t$. 
Next, we define the true potential function as

$$
V_{T}(\bar{q})=\Omega^{2}(\bar{q})+E
$$

where $V_{T}: \mathbb{C} \rightarrow \mathbb{C}$ is written in terms of the function $\Omega: \mathbb{C} \rightarrow \mathbb{C}$ for convenience. The complex Hamiltonian of $V_{T}$ is given by

$$
\begin{gathered}
H=\frac{p^{2}}{2 m}+V_{T} \\
H=\frac{p^{2}}{2 m}+\Omega^{2}+E
\end{gathered}
$$

Setting $H=E$ (from eq. 11) gives

$$
\begin{gathered}
E=\frac{p^{2}}{2 m}+\Omega^{2}+E \\
p=i \sqrt{2 m} \Omega
\end{gathered}
$$

Therefore, the derivative of $p$ with respect to $\bar{q}$ is given by

$$
\frac{d p}{d \bar{q}}=i \sqrt{2 m} \frac{d \Omega}{d \bar{q}}
$$

Now, in order to write eq. 32 as a second order linear partial differential equation, we make use of eq. 26 , and get

$$
\begin{gathered}
-i \hbar \frac{\partial}{\partial \bar{q}}\left(-i \hbar \frac{\partial \Psi}{\partial \bar{q}}\right)=-i \hbar \frac{\partial}{\partial \bar{q}}(p \Psi) \\
-\hbar^{2} \frac{\partial^{2} \Psi}{\partial \bar{q}^{2}}=-i \hbar \frac{\partial p}{\partial \bar{q}} \Psi-i \hbar \frac{\partial \Psi}{\partial \bar{q}} p \\
-\hbar^{2} \frac{\partial^{2} \Psi}{\partial \bar{q}^{2}}=-i \hbar \frac{d p}{d \bar{q}} \Psi+p^{2} \Psi \\
p^{2} \Psi=-\hbar^{2} \frac{\partial^{2} \Psi}{\partial \bar{q}^{2}}+i \hbar \frac{d p}{d \bar{q}} \Psi
\end{gathered}
$$

Multiplying eq. 32 by $\Psi$ gives

$$
H \Psi=\frac{p^{2}}{2 m} \Psi+\Omega^{2} \Psi+E \Psi
$$

Substituting eq. 35 into eq. 36 first, and then making use of eq. 34 , leads to

$$
\begin{gathered}
H \Psi=-\frac{\hbar^{2}}{2 m} \frac{\partial^{2} \Psi}{\partial \bar{q}^{2}}+\frac{i \hbar}{2 m} \frac{d p}{d \bar{q}} \Psi+\Omega^{2} \Psi+E \Psi \\
H \Psi=-\frac{\hbar^{2}}{2 m} \frac{\partial^{2} \Psi}{\partial \bar{q}^{2}}-\frac{\hbar}{\sqrt{2 m}} \frac{d \Omega}{d \bar{q}} \Psi+\Omega^{2} \Psi+E \Psi
\end{gathered}
$$

$$
H \Psi=-\frac{\hbar^{2}}{2 m} \frac{\partial^{2} \Psi}{\partial \bar{q}^{2}}+V \Psi
$$

where we defined the function $V: \mathbb{C} \rightarrow \mathbb{C}$ as

$$
V=-\frac{\hbar}{\sqrt{2 m}} \frac{d \Omega}{d \bar{q}}+\Omega^{2}+E
$$

Applying the second condition of the observer's assumption (eq. 25) to eq. 37 gives

$$
H \Psi=-\frac{\hbar^{2}}{2 m} \frac{\partial^{2} \Psi}{\partial x^{2}}+V \Psi
$$

Equation 39 can be seen as the definition of the Hamiltonian operator. By setting $H=E$ (from eq. 11) we get

$$
E \Psi=-\frac{\hbar^{2}}{2 m} \frac{\partial^{2} \Psi}{\partial x^{2}}+V \Psi
$$

This is the one-dimensional time independent Schrödinger equation. Finally, from eq. 30 and eq. 40 we have

$$
i \hbar \frac{\partial \Psi}{\partial t}=-\frac{\hbar^{2}}{2 m} \frac{\partial^{2} \Psi}{\partial x^{2}}+V \Psi
$$

which is the one-dimensional time dependent Schrödinger equation. The one-dimensional equations corresponding to the $y-w$ and $z-w$ planes can be derived from a similar analysis. The three-dimensional Schrödinger equation can then be obtained by combining them.

As a final note, if a potential function $V$, can be written in terms of $\Omega$ according to the definition given by eq. 38 , then it can be shown that the exact solution to the lowest energy level of Schrödinger's equation will be

$$
\Psi(x, t)=e^{-\sqrt{2 m} \int \Omega d x / \hbar} e^{-i E t / \hbar}
$$

The derivation of this equation is given in the Appendix.

\section{Conclusion} formulated an interpretation of the mathematical formalism of quantum mechanics based on the hypothesis 
that physical space is a four-dimensional incompressible inviscid fluid that undergoes irrotational flow. In order to prove this interpretation is mathematically valid, we derived the one-dimensional Schrödinger equation from the equations for two-dimensional incompressible irrotational inviscid fluid flow (via the complex conjugate Hamilton-Jacobi equation). A few additional arguments that support our main thesis are the following:

- In quantum mechanics, some eigenvectors are complex (e.g., the momentum eigenvectors), while others are purely real (e.g., the harmonic oscillator energy eigenvectors). This suggests the wave function could be expressed as a complex function evaluated at a value that forces its imaginary component to be zero under some conditions. The definition of the wave function given by eq. 24, together with the second mathematical condition of the observer's assumption (specified via eq. 25), satisfy that requirement.

- The wave function of Schrödinger's formulation is differentiable (we consider the Dirac delta function as a special case). Therefore, Hamilton's complex principal function in eq. 24 must be analytic. This means the Cauchy-Riemann conditions corresponding to $\bar{q}$ are satisfied. It follows that the flow describing the system is incompressible and irrotational.

- Schrödinger's equation deals only with conservative forces. Hence, if physical space is indeed a fluid, it must be inviscid.

The Four-Dimensional Fluid Space Interpretation of Quantum Mechanics is a realistic deterministic local hidden-variable theory. Bell's theorem proves local hidden-variable theories cannot replicate the same results of quantum mechanics. This apparent conflict needs to be addressed, so we proceed to do so. Bell's theorem assumes the mathematical formalism of quantum mechanics describes particles. For that reason, the probabilities used in Bell's theorem do not take into account superposition. We claim eigenvectors in quantum mechanics are actually describing incompressible irrotational inviscid fluid flows. Indirect and incomplete information about the particle is obtained from the streamlines of these flows. Because we are dealing with a particle-fluid system, superposition of flows needs to be taken into account when calculating the probabilities of each quantum state. Furthermore, it can also be argued that the interpretation formulated here is a counter example to Bell's theorem. In a future paper, we intend to provide a rigorous proof based on these arguments.

\section{DEDICATION}

$\mathrm{T}$ HE research presented here is dedicated to the memory of my father, Dr. Lorenzo León Callender López, who always supported me and was there for me. Without him, this research would not have been possible.

\section{APPENDIX}

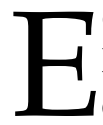
QUATION 42 is the exact solution to the lowest energy level of Schrödinger's equation. That result is derived in this Appendix. We start by integrating eq. 17 to get

$$
W=\int p d \bar{q}
$$

Then, we write eq. 14 in terms of eq. 43 . This gives

$$
S(\bar{q}, t)=\int p d \bar{q}-E t
$$

After that, we substitute eq. 44 into eq. 24

$$
\Psi(\bar{q}, t)=e^{i \int p d \bar{q} / \hbar} e^{-i E t / \hbar}
$$

and use eq. 33 to write eq. 45 in terms of $\Omega$.

$$
\Psi(\bar{q}, t)=e^{-\sqrt{2 m} \int \Omega d \bar{q} / \hbar} e^{-i E t / \hbar}
$$

Finally, we apply the second mathematical condition of the observer's assumption (eq. 25) to obtain

$$
\Psi(x, t)=e^{-\sqrt{2 m} \int \Omega d x / \hbar} e^{-i E t / \hbar}
$$

which is the desired result. The corresponding energy eigenvalue can be obtained by applying eq. 25 to eq. 38 , and solving for $E$. 


\section{REFERENCES}

[1] Jammer, Max. The Philosophy of Quantum Mechanics: The Interpretations of QM in Historical Perspective. USA: John Wiley and Sons; 1974.

[2] Nordström, Gunnar. Uber die Moglichkeit, das Elektromagnetische Feld und das Gravitationsfeld $\mathrm{Zu}$ Vereinigen. (German) [On the Possibility of Unifying the Electromagnetic and the Gravitational Fields]. Phys. Z. 1914; 15: 504-506

[3] Kaluza, Theodor. Zum Unitätsproblem der Physik. (German) [On the Unification Problem in Physics]. Sitzungsberichte der Königlich Preußischen Akademie der Wissenschaften (Berlin). 1921; 1921: 966-972

[4] Madelung, Erwin. Quantentheorie in Hydrodynamischer Form. (German) [Quantum Theory in Hydrodynamical Form]. Zeitschrift für Physik. 1927; 40: 322-326 